\title{
Voyager 2 Observations of the Anisotropy of Anomalous Cosmic Rays in the Heliosheath
}

\author{
A. C. Cummings ${ }^{* a}$, E. C. Stone ${ }^{a}$, B. C. Heikkila ${ }^{b}$, N. Lal ${ }^{b}$, J. D. Richardson ${ }^{c}$ \\ ${ }^{a}$ Caltech, Pasadena $C A$ \\ ${ }^{b}$ Goddard Space Flight Center, Greenbelt, $M D$ \\ ${ }^{c}$ MIT, Boston, $M A$ \\ E-mail: acedsrl.caltech.edu, ecsesrl.caltech.edu, \\ bryant.heikkila@gmail.com, voycrsegmail.com, jdr@space.mit.edu
}

\begin{abstract}
We have analyzed data collected from the Cosmic Ray Subsystem instrument [1] on the Voyager 2 (V2) spacecraft during calibration manuevers for the magnetic field instrument while the spacecraft was located in the inner heliosheath of the heliosphere. We find that there is a diffusive anisotropy in the intensity of $\sim 0.5-35 \mathrm{MeV}$ particles (mostly protons) that indicates that there exists a diffusive flow of these particles from the flank or tail of the heliosphere towards the nose and is slightly equatorward as well. This work is consistent with the findings of an earlier, similar, but more restricted study [2]. Depending on which part of the energy range is the main contributor to the observed diffusive anisotropy, these results may indicate that the acceleration site of anomalous cosmic rays is back towards the flank or tail of the solar wind termination shock.
\end{abstract}

36th International Cosmic Ray Conference -ICRC2019-

July 24th - August 1st, 2019

Madison, WI, U.S.A.

* Speaker. 


\section{Introduction}

When the Voyager spacecraft made their crossings of the solar wind termination shock, neither observed the shape of the energy spectrum of anomalous cosmic rays (ACRs) from diffusive shock acceleration

(DSA)

$[3,4]$, which at least at the time of the Voyager 1 (V1) crossing in December 2004 was the expected shape. Several theories have emerged since then to propose other acceleration sites and mechanisms.

One possibility that emerged soon after the V1 crossing was that DSA might still be the acceleration mechanism but that it occured further back along the tail or flank of a blunt termination shock rather than where V1 had crossed [5, 6, 7]. The main energization would take place there where the magnetic field lines would stay in contact with the shock for a longer time, allowing acceleration to higher energies. Also, because of the angle of the field to the shock back along the flank, lower injection

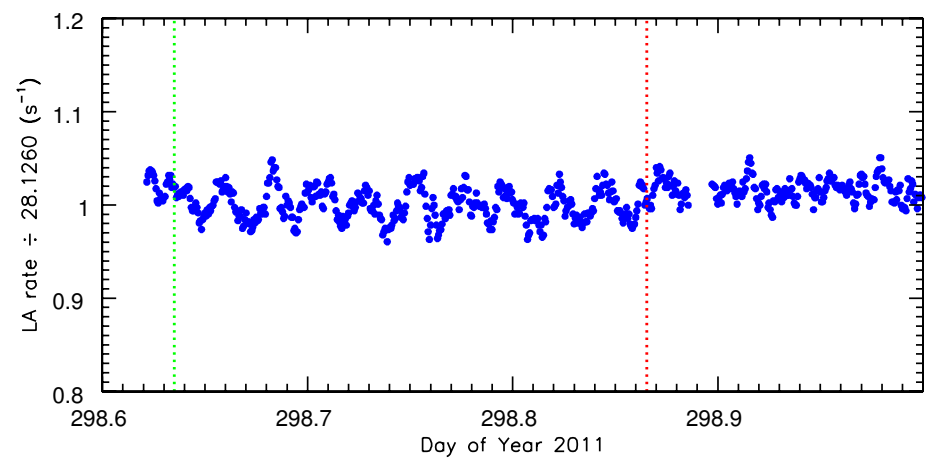

Figure 1: Counting rate of mostly protons with $\sim 0.5-35 \mathrm{MeV}$ from the LET A L1 detector (in anti-coincidence with the L4 detector [1]) during ten spacecraft rolls on day 298 of 2011. The symbols are 5-point moving averages, where a point represents data collected over a 48 -second time period. The vertical green line marks the start of the roll and the vertical red line marks the end of the roll.

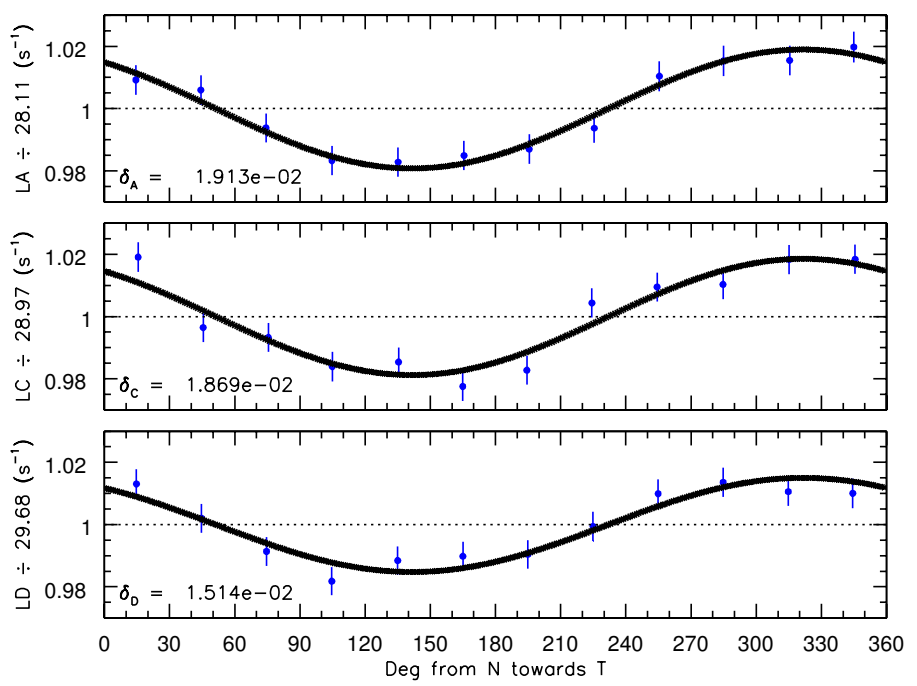

Figure 2: (top panel) Data from Figure 1 binned by angle in $30^{\circ}$ bins from the $\mathrm{N}$ axis towards the $\mathrm{T}$ axis. The solid line represents the fit to a 1 st-order anisotropy function as described in the text. The value of the anisotropy amplitude for the LET A data, $\delta_{A}$, derived from the fit is shown in the figure. (middle and bottom panels) Same as top panel except for LETs C and D, respectively. 
energies would be allowed.

The observed evolution of the ACR energy spectra supported this view, as the energy spectra unfolded to the expected shape from DSA as the Voyagers penetrated further into the inner heliosheath [8]. However, such spectral evolution would also be expected if the source of the particles were further out in the heliosphere in front of the Voyagers as predicted by other models $[9,10,11]$.

Another aspect

of having the ACRs accelerated in the flank or tail of the heliosphere and diffusing towards the nose is that there should be an anisotropy associated with the flow. Stone et al., 2017 [2], investigated that possibility based on observations of particle flow anisotropies made during rolls of the V2 spaceraft about the axis pointed towards Earth that occur typically every few months in order to provide calibration of the magnetometer. They concluded that there is likely a diffusive flow of ACRs from the flank towards the nose based on rolls from mid-2011 through 2015, since attempts to account for the solar wind $\mathrm{T}$ and $\mathrm{N}$ components ${ }^{1}$ by way of the Compton-Getting effect [13] failed, primarily in the T component. In this paper we add additional rolls that occurred before and after the periods analyzed in that report and quantify the diffusive components of the flow.

\footnotetext{
${ }^{1}$ The RTN coordinate system is spacecraft centered with R pointing radially away from the Sun, T is parallel to the solar equator and in the direction of the Sun's rotation, and N completes the right-handed system[12].
} 


\section{Observations}

The Voyager spacecraft are normally three-axis stabilized, but periodically they are rolled about the $\mathrm{R}$ axis to help calibrate the magnetometer. Prior to 2016, the rolls consisted of 10 revolutions about the $\mathrm{R}$ axis. However, due to power issues there were no rolls in 2016 and the ones in 2017 and 2018 were limited to 1 or 2 revolutions. While the larger number of revolutions is helpful for statistical purposes, we find we can still use the rolls with the smaller number of revolutions to fairly accurately determine the anisotropy components in the N-T plane.

In Figure 1 we show the counting rate from the Low-Energy Telescope (LET) A L1 detector (mostly protons with $\sim 0.5$ $35 \mathrm{MeV}$ ) during a typical 10-revolution roll maneuver in 2011. The periodic variation is consistent with a firstorder anisotropy with a period corresponding to the 2000 second duration of one $360^{\circ}$ revolution.

In Figure 2 we show the data from all three telescopes available, LETs A, C, and D, from day 298 of 2011, along with the result of a least-squares fit to a first-order anisotropy function to the rates in each telescope: $J_{A}=J_{0}(1+\vec{\delta} \cdot \vec{A})$, $J_{C}=k_{C} J_{0}(1+\vec{\delta} \cdot \vec{C})$, and $J_{D}=k_{D} J_{0}(1+\vec{\delta} \cdot \vec{D})$,
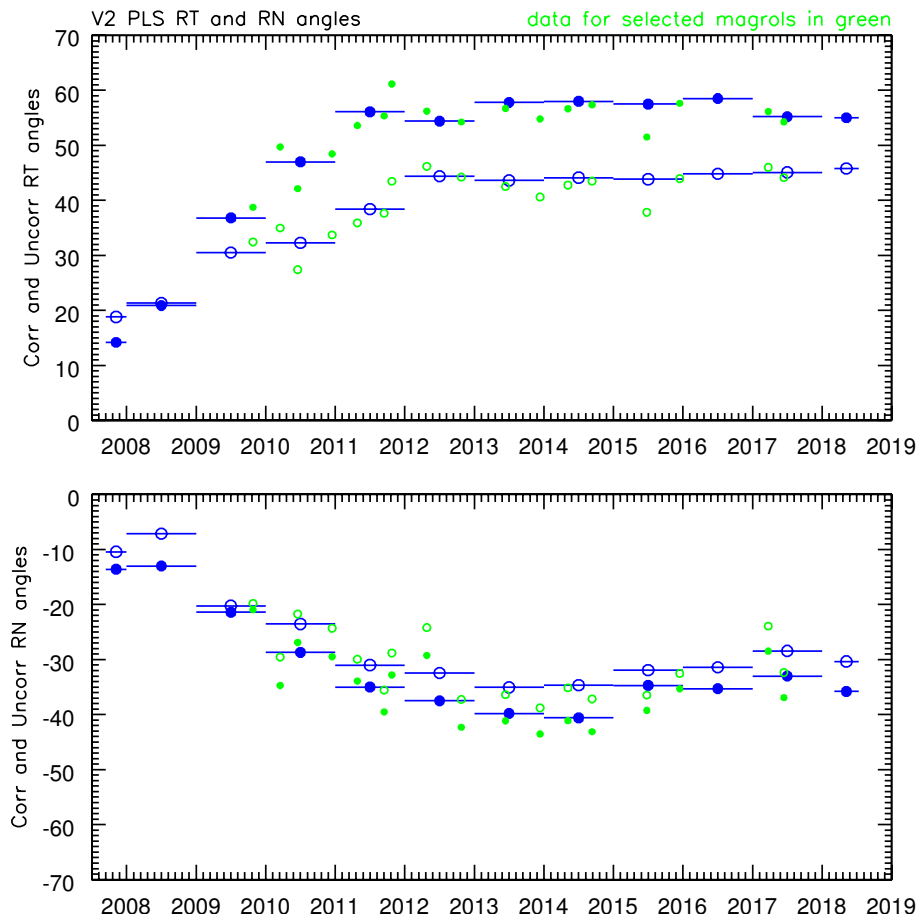

Figure 4: (top panel) Uncorrected angle in the R-T plane of the solar wind flow (open symbols) together with the corrected angle (solid symbols) vs time. The larger, blue symbols represent long-term averages where the correction can be accurately estimated. The smaller green symbols represent data from the days of selected magrols; the selection and correction procedures are described in the text. (bottom panel) Same as top panel except for angle in the R-N plane.

where $\vec{A}$, for example, refers to the unit vector that represents the boresight of LET A, and $k_{C}$ and $k_{D}$ represent normalization factors. The data are binned by angle in the N-T plane and thus the equations that are used in the fitting are written to accommodate that situation. For example, let $\beta_{A}$ represent the angle of the LET A boresight from $\mathrm{N}$ towards $\mathrm{T}$ in the $\mathrm{N}-\mathrm{T}$ plane. This angle advances $8.64^{\circ}$ for each $48 \mathrm{~s}$ data point and is known based on the original boresight vector, the start time of the roll, and the roll rate. Let $\theta_{A}$ be the fixed angle of the LET A boresight vector from the $\mathrm{R}$ axis. Then, the components of the LET A boresight vector are $A_{R}=\cos \theta_{A}$, 
$A_{T}=\sin \theta_{A} \sin \beta_{A}$, and $A_{N}=\sin \theta_{A} \cos \beta_{A}$. The fit parameters are $J_{0}, k_{C}, k_{D}$, and the $\mathrm{N}$ and $\mathrm{T}$ components of the anisotropy vector, $\vec{\delta}$. The R component of $\vec{\delta}$ is not fit but is taken to be that calculated from the Compton-Getting effect (described later) based on the measured $\mathrm{R}$ component of the solar wind velocity (corrected into the spacecraft frame of rest). To test the sensitivity of the result to the value of $\delta_{R}$ used, we experimented with the fits to the roll on 2015/349 and found that if $\delta_{R}$ was multiplied by factors of 0.5 and 2 , the $\delta_{T}$ and $\delta_{N}$ values changed by less than $0.5 \%$.

Note that from
the equations used in the fit, the rate is maximum for positive $\vec{\delta}$, so that $\vec{\delta}$ points towards the direction from which the flow is coming. In Figure 3, we have multiplied the components by a factor of $-1.193^{2}$ and labeled them as "true" to account both for the wide field-of-view of the telescope $\left(120^{\circ}\right)$ and to represent the direction to which the particles are flowing.

Figure 3 shows the results for $J_{0}$ and the true $\mathrm{T}$ and $\mathrm{N}$ components of $\vec{\delta}$ for all 55 magrols that occurred between the V2 crossing of the temination shock on 30 August 2007 and its crossing of the
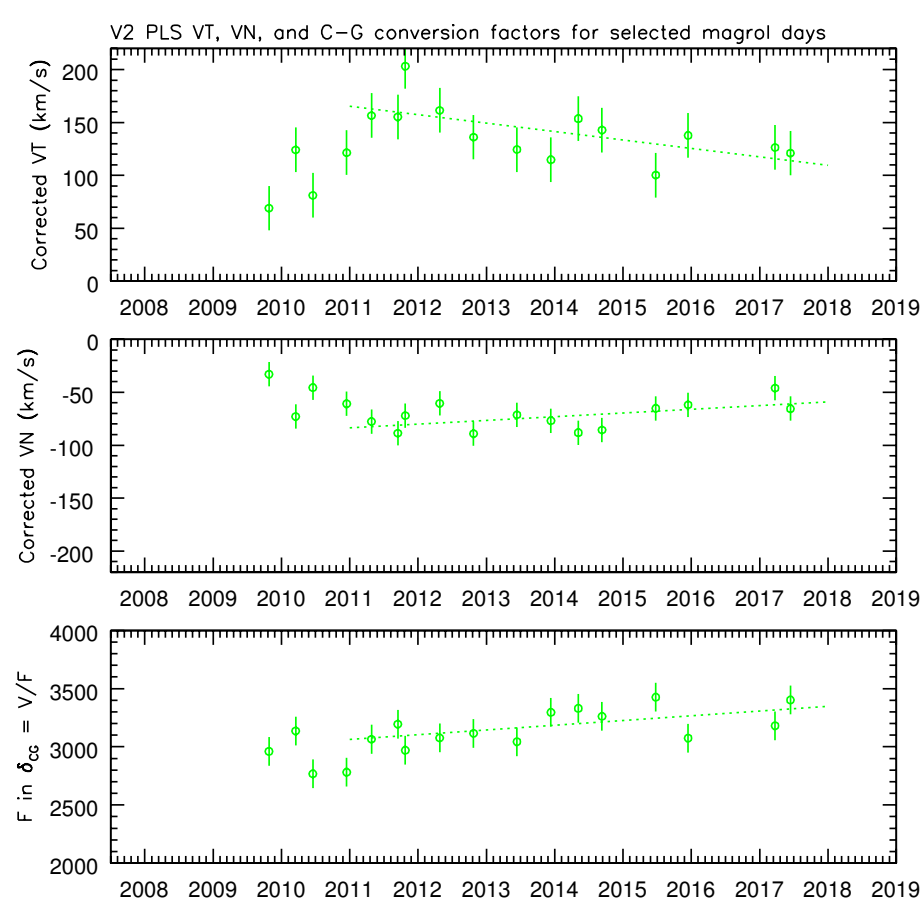

Figure 5: (top panel) Corrected T-component of solar wind velocity for selected magrols. (middle panel) Same as top panel except for N-component of solar wind velocity. (bottom panel) Factor F that converts solar wind speed to the convective Compton-Getting anisotropy. The uncertainties in each panel were assigned such that the linear fits shown resulted in a reduced $\chi^{2}$ of 1.0 .

heliopause on 5 November 2018. The 9 rolls that were judged not to yield good fits by the Q statistic test are shown as red symbols in Figure $3 .^{3}$

Since we are interested in the flows during quiet, undisturbed periods, we examined the counting rates of the L1 detectors in the three telescopes and labeled as transients any roll days that appeared to be affected by disturbances. Fourteen of these were identified and are shown by the blue symbols. The 32 remaining rolls, shown by the green symbols, were selected for further

\footnotetext{
${ }^{2}$ The calculations that led to the factor 1.193 are based on Space Radiation Lab Internal Report 84 by Gehrels and Chenette, 1981, which is available on request from the author.

${ }^{3}$ The Q statistic is the complement of the chi-square probability function ([14], equation 6.2.19) and the fit was judged to be good if its value was within 0.05 and 0.999 .
} 
analysis to determine the $\mathrm{N}$ and $\mathrm{T}$ components of the diffusive anisotropy.

The diffusive anisotropy is derived from the observed anisotropy by subtracting the convective anisotropy due to the Compton-Getting (C-G) effect [13]. The C-G anisotropy, $\overrightarrow{\delta_{C G}}$, is given by

$$
\overrightarrow{\delta_{C G}}=<(2-2 \gamma) / v>\vec{V}
$$

where $v$ is particle speed, $\vec{V}$ is the solar wind velocity, and $\gamma$ is the power-law index in the differential energy spectrum, $\mathrm{dJ} / \mathrm{dE} \sim \mathrm{E}^{\gamma}$, and the brackets $<>$ denote the average over the energy spectrum of the enclosed quantity. We have fit the energy spectra data to a 4-power-law function for each roll day and then employed a Monte-Carlo simulation using that function from 0.4-40 MeV to select particles and input them onto the telescope. The result of the simulation is the coefficient of $\vec{V}$ in equation 2.1 .

We need to apply that coefficient to the components of the solar wind velocity to obtain the components of $\overrightarrow{\delta_{C G}}$. As noted in [15], as the solar wind in the inner heliosheath turns away from the radial direction to flow back down the tail of the heliosphere, the Plasma Science instrument (PLS) response affects the measurement. If one integrates the data over long enough periods, the flow angles in the $\mathrm{R}-\mathrm{T}$ and R-N planes can be corrected. Once the corrected angles are obtained, the corrected $\mathrm{N}$ and $\mathrm{T}$ components of the solar wind velocity can be calculated from the radial component
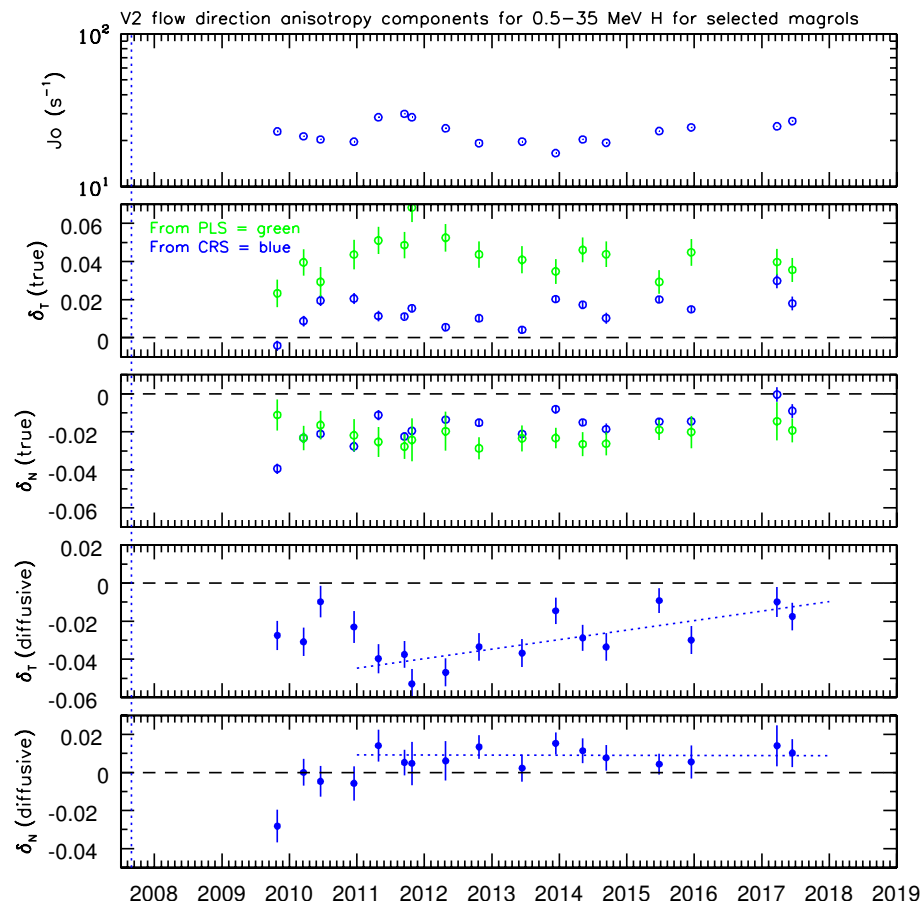

Figure 6: (top three panels) Similar to Figure 3 except only for the final 17 selected roll days. In addition, the Compton-Getting components (labeled "From PLS = green") are shown and based on the corrected solar wind speeds and the energy spectra for the roll days. (bottom two panels) $\mathrm{T}$ and $\mathrm{N}$ components of the diffusive anisotropy vectors as described in the text. Dotted lines represent linear fits to the data from 2011.0 forward. times the tangent of the corrected angle.

There are significant fluctuations in the direction of plasma flow on the scales of days or less [15], and since it is not possible to make the angle corrections for the roll days individually, we selected roll days for further analysis that had values of the RT and RN angles that were consistent with the uncorrected long-term averages. We first determined two distributions of differences of 
long-term and roll-day angles for all 55 rolls, one disribution for the RT angle differences and one for the RN angle differences. We eliminated those roll days that had angle differences in the tails of either distribution, with the tail being defined as outside 1 standard deviation of the average of the distribution. That selection, in conjunction with the previous selection criteria, left 17 rolls for the derivation of the $\mathrm{R}$ and $\mathrm{T}$ components of the diffusive anisotropy.

Figure 4 shows the uncorrected and corrected RT and RN angles for both the long-term average periods and the 17 selected roll days. The corrected angle for a roll day was the result of adding the same amount of correction as for the long-term average in which time period the roll occurred.

Figure 5 shows the corrected $\mathrm{T}$ and $\mathrm{N}$ components of the solar wind velocity and the divisor $\mathrm{F}$ that converts solar wind velocity into a Compton-Getting anisotropy.

\section{Results and Summary}

The bottom two panels of Figure 6 show the results of this investigation, which are the T and $\mathrm{N}$ components of the diffusive anisotropy of protons with $0.5-35 \mathrm{MeV}$. For the diffusive $\delta_{T}$, there appears to be a linear trend towards zero after 2011.0. For diffusive $\delta_{N}$, there is no trend after 2011.0. For most of the time, the flow is mainly in the -T direction, which would indicate a flow from the flank or tail of the heliosphere towards the nose. There is a small upward angle to the flow towards the helioequator.

The diffusive anisotropy depends on particle speed, $v$, the diffusion tensor, $K$, and the gradient of the number density per unit energy, $U[13]$ :

$$
\overrightarrow{\delta_{\text {diff }}}=-(3 / v) K \cdot \nabla U / U
$$

A typical value of $\left\langle 1 / v>\right.$ (from the roll on 2015/349) is $6.36 \times 10^{-5} \mathrm{~s} \mathrm{~km}^{-1}$. The measurements reported here thus place constraints on $K$ and the gradient of $U$.

We note that the proton energy range investigated here, $0.5-35 \mathrm{MeV}$, spans the range between low-energy termination shock particles (TSPs)[3] and ACRs. These two components of the energy spectrum in the heliosheath had very different spectral evolution histories. The TSP intensities stayed rather steady after the termination shock crossings, whereas the ACRs showed a modulated spectrum at the shock crossing, which gradually evolved to an ummodulated shape over time (see Figure 3 of Cummings and Stone, 2008[16]). This behavior suggests that the TSPs were locally accelerated at shock crossing, whereas the ACRs were accelerated at a remote location. Thus, the interpretation of the observed diffusive anisotropy of the $0.5-35 \mathrm{MeV}$ protons depends on which part of the energy spectrum is mainly responsible for it. If the main contributor is in the ACR part of the energy spectrum, then these results suggest that the acceleration site of ACRs is along the flank or tail of the solar wind termination shock. If the TSPs dominate the anisotropy, then other sites for the acceleration of ACRs are posasible. Further modeling is needed to investigate these results.

\section{Acknowledgments}

Voyager data analysis is supported by NASA Grant NNN12AA01C. 


\section{References}

[1] E. C. Stone, R. E. Vogt, F. B. McDonald, B. J. Teegarden, J. H. Trainor, J. R. Jokipii, and W. R. Webber, Cosmic Ray Investigation for the Voyager Missions: Energetic Particle Studies in the Outer Heliosphere - and Beyond, Space Sci. Rev. 21 (1977) 355-376.

[2] E. Stone, A. C. Cummings, B. C. Heikkila, N. Lal, and W. R. Webber, The Anisotropy of Anomalous Cosmic Rays Observed by Voyager 2 in the Heliosheath, International Cosmic Ray Conference 301 (Jan, 2017) 57.

[3] E. C. Stone, A. C. Cummings, F. B. McDonald, B. C. Heikkila, N. Lal, and W. R. Webber, Voyager 1 Explores the Termination Shock Region and the Heliosheath Beyond, Science 309 (Sept., 2005) 2017-2020.

[4] E. C. Stone, A. C. Cummings, F. B. McDonald, B. C. Heikkila, N. Lal, and W. R. Webber, An asymmetric solar wind termination shock, Nature 454 (July, 2008) 71-74.

[5] D. J. McComas and N. A. Schwadron, An explanation of the Voyager paradox: Particle acceleration at a blunt termination shock, Geophys. Res. Lett. 33 (Feb., 2006) 4102.

[6] J. Kóta, Anomalous cosmic rays at a blunt termination shock, International Cosmic Ray Conference 1 (2008) 853-856.

[7] J. Kóta and J. R. Jokipii, Implications of the Voyager-1 particle spectra for acceleration at the termination shock, in Physics of the Inner Heliosheath (J. Heerikhuisen, V. Florinski, G. P. Zank, and N. V. Pogorelov, eds.), vol. 858 of American Institute of Physics Conference Series, pp. 171-176, Sept., 2006.

[8] A. C. Cummings, E. C. Stone, F. B. McDonald, B. C. Heikkila, N. Lal, and W. R. Webber, Anomalous Cosmic Rays in the Heliosheath, in American Institute of Physics Conference Series (G. Li, Q. Hu, O. Verkhoglyadova, G. P. Zank, R. P. Lin, and J. Luhmann, eds.), vol. 1039, pp. 343-348, Aug, 2008.

[9] A. Lazarian and M. Opher, A Model of Acceleration of Anomalous Cosmic Rays by Reconnection in the Heliosheath, Astrophys. $\mathbf{7 0 3}$ (Sept., 2009) 8-21, [arXiv: 0905.1120].

[10] J. F. Drake, M. Opher, M. Swisdak, and J. N. Chamoun, A Magnetic Reconnection Mechanism for the Generation of Anomalous Cosmic Rays, Astrophys. J 709 (Feb, 2010) 963-974, [arXiv:0911.3098].

[11] L. A. Fisk and G. Gloeckler, The acceleration of Anomalous Cosmic Rays by stochastic acceleration in the heliosheath, Advances in Space Research 43 (May, 2009) 1471-1478.

[12] L. F. Burlaga, Magnetohydrodynamic Processes in the Outer Heliosphere, Space Science Reviews 39 (Dec, 1984) 255-316.

[13] M. A. Forman, The Compton-Getting effect for cosmic-ray particles and photons and the Lorentz-invariance of distribution functions, Planet. Space Sci. 18 (Jan., 1970) 25.

[14] W. H. Press, S. A. Teukolsky, W. T. Vetterling, and B. P. Flannery, Numerical recipes in FORTRAN. The art of scientific computing. 1992.

[15] J. D. Richardson and R. B. Decker, Voyager 2 Observations of Plasmas and Flows out to 104 AU, Astrophys. J. 792 (Sept., 2014) 126.

[16] A. C. Cummings and E. C. Stone, Possible role of transients on the energy spectra of energetic particles at the solar wind termination shock, in International Cosmic Ray Conference, vol. 1 of International Cosmic Ray Conference, pp. 827-830, Jan, 2008. 